\title{
Estudio teórico de una nueva estrategia terapéutica en aterosclerosis basada en la inhibición del inflamasoma NLRP3.
}

\author{
Rodrigo Damián García ${ }^{a}$, Paula Gutiérrez ${ }^{b}$, Marina Higuerac ${ }^{c}$ Paula Moreno ${ }^{d}$, \\ Daniela Paola Poullion ${ }^{\mathrm{e}}$ \\ Unidad de Bioquímica y Biología Molecular, Departamento de Biología de Sistemas, Facultad de Medicina y Ciencias \\ de la Salud, Universidad de Alcalá, 28871 Alcalá de Henares, Madrid, España.
}

a. rodridg@hotmail.com b. paugugar@gmail.com c. marinahigueragarcia@gmail.com d. pmoreno.bm@gmail.com e. danypoullion@gmail.com

IV Congreso de Señalización Celular, SECUAH 2019.

20-22 de marzo, 2019. Universidad de Alcalá. Alcalá de Henares, Madrid. España

Sesión de paneles.

Palabras clave: Aterosclerosis; HTS; Inflamasoma; NLRP3; IL-1 $\beta$

\section{Resumen}

La aterosclerosis es una de las enfermedades cardiovasculares de mayor prevalencia a nivel mundial. Históricamente su fisiopatología se ha centrado en el papel de los lípidos y su rol en el desarrollo de la placa de ateroma. En los últimos años, diversos estudios han demostrado la gran relevancia del componente inflamatorio en la patogénesis de la aterosclerosis, en concreto, la Interleucina 1 Beta (IL-1 $\beta$ ) ha demostrado tener un papel fundamental en esta patología. Basándonos en esta evidencia, desarrollamos un estudio teórico de las posibles vías inflamatorias relacionadas con IL-1ß implicadas en la inflamación vascular, valorando diversas alternativas como eventuales dianas terapéuticas. De entre ellas, destacamos el complejo inflamasoma Receptor tipo NOD asociado a proteína 3 (NLRP3), cuya asociación conduce a la activación y liberación de IL-1 $\beta$ e interleucina 18 (IL-18), ambas citoquinas proaterogénicas. Para la obtención de posibles inhibidores del inflamasoma NLRP3 a partir de nuestra quimioteca de más de 2 millones de compuestos, se propuso un análisis de tipo High Throughput Screening (HTS) en el que se empleó una técnica de tipo transferencia de energía basada en resonancia de fluorescencia (FRET). De este análisis FRET se obtuvieron aquellos inhibidores específicos de la asociación entre los componentes del inflamasoma NLRP3, denominados hits. Esta búsqueda se refinó mediante ensayos in vitro con células THP1 deficientes de NLRP3. Se obtuvieron así aquellos compuestos de baja toxicidad que antagonizaban el inflamasoma NLRP3, produciendo una menor liberación de IL-1 e IL-18, considerados nuestros leads o cabezas de serie. Finalmente, para la evaluación de los candidatos leads se diseñó un estudio in vivo con ratones ApoE ${ }^{(-)}$con el objetivo de confirmar su eficacia y potencia farmacológica.

Cita: García, Rodrigo Damián; Gutiérrez, Paula; Higuera, Marina; Moreno, Paula; Poullion, Daniela Paola (2019) Estudio teórico de una nueva estrategia terapéutica en aterosclerosis basada en la inhibición del inflamasoma NLRP3. Actas del IV Congreso de Señalización Celular, SECUAH 2019. 20-22 de marzo, 2019. Universidad de Alcalá. Alcalá de Henares, Madrid. España. Sesión de paneles. dianas 8 (1): e201903fa02. ISSN 1886-8746 (electronic) journal.dianas.e201903fa02 http://www3.uah.es/dianas?e201903fa02. URI http://hdl.handle.net/10017/15181

Copyright: @ García RD, Gutiérrez P, Higuera M, Moreno P, Poullion DP. Algunos derechos reservados. Este es un artículo open-access distribuido bajo los términos de una licencia de Creative Commons ReconocimientoNoComercial-SinObraDerivada 4.0 Internacional. http://creativecommons.org/licenses/by-nc-nd/4.0/

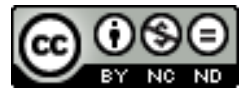

\section{Introducción}

La enfermedad cardiovascular es una de las principales causas de muerte en el mundo occidental. Según datos de la Organización Mundial de la Salud (OMS), en el año 2015 alrededor de 7.4 millones de personas murieron a causa de una enfermedad coronaria aterosclerótica y, se estima que estas cifras se incrementarán en los próximos años. [1]

Evidencias científicas recientes proponen a la inflamación vascular como uno de los mecanismos principales en el desarrollo de la aterosclerosis. Durante años, los lípidos han jugado un papel fundamental en la escena de la fisiopatología de la enfermedad aterosclerótica, de manera que no es de extrañarse que muchos de los estudios clínicos hayan centrado su atención en moléculas que reducen los niveles de colesterol con el fin de minimizar el riesgo cardiovascular. Sin embargo, en la mayoría de estos ensayos clínicos, tanto retrospectivos como prospectivos, la inhibición de la HMG-CoA reductasa se tradujo en una reducción de las tasas de eventos vasculares entre los individuos con una mayor tasa inflamatoria frente a los que no la presentaban. Entre estos estudios destaca el estudio CARE con pravastatina [2], los estudios AFCAPS / TexCAPS con lovastatina [3], o los ensayos JUPITER y SATURN con rosuvastatina [4 - 5]. En el caso del ensayo JUPITER, un estudio que sin dudas cambio el 
rumbo de la cardiología clínica, el riesgo absoluto cardiovascular aumentó con los niveles crecientes de proteína C Reactiva ultrasensible (PCR-us) independientemente del valor inicial de colesterol-LDL. Además, la magnitud de la reducción del riesgo relativo observado en el estudio JUPITER (que incluyó información sobre inflamación) fue mayor que la observada previamente en todos los ensayos con estatinas, que sólo se basaron en el control de la hiperlipidemia [4]. Siguiendo esta línea, varios ensayos clínicos como PROVE IT [6], A to Z [7], REVERSAL [8] o SATURN [5] coinciden en señalar que la disminución de los niveles de PCR-us contribuyen en la reducción de eventos de manera análoga a la consecución de los bajos niveles de colesterol-LDL. A pesar de ello, este tipo de análisis en los ensayos con estatinas no permitió establecer si la reducción exclusiva de la inflamación disminuiría el riesgo cardiovascular, debido a la capacidad de las estatinas de modificar tanto el perfil inflamatorio como los niveles de lípidos. Recientemente, con los primeros resultados del estudio CANTOS [9] se pudo probar la hipótesis del gatillo inflamatorio en la enfermedad aterotrombótica. En este estudio, el empleo de Canakinumab, un anticuerpo monoclonal humano anti IL-1 $\beta$ con efectos antiinflamatorios aprobado para su uso en enfermedades reumatológicas, logró reducir de forma significativa la tasa de eventos cardiovasculares independientemente de la reducción en el nivel lipídico.

La acumulación de datos clínicos, como los citados anteriormente, nos proporciona la base científica para formular una hipótesis centrada en la búsqueda de nuevos agentes antiinflamatorios específicos destinados a modificar el curso de la aterosclerosis. Un enfoque particularmente prometedor sería la inhibición del complejo NLRP3, una pieza clave para comprender el fenómeno inflamatorio generado en esta enfermedad. [10 - 11].

\section{Papel del inflamasoma}

Un inflamasoma es un complejo citoplasmático formado por varias proteínas que actúa como receptor de reconocimiento de patrones (PRR). Su función es el reconocimiento de señales de peligro, incluidos los patrones moleculares asociados a patógenos (PAMP) y los patrones moleculares asociados a daños / peligros (DAMP). Este tipo de complejos se expresa en las células involucradas en el sistema inmunitario innato, como los macrófagos, los neutrófilos y las células dendríticas. Los PRR se clasifican en varios grupos según su estructura, función y localización conservadas. En el caso de los NLR, éstos exhiben una localización intracelular [12]. En relación con su función celular, Tschopp y su grupo de investigación utilizaron el término inflamasoma para definir aquella plataforma necesaria para la activación de la caspasa-1 [13]. La caspasa-1 es la enzima encargada de la escisión y activación de la IL-1 $\beta$, citoquina conocida por su rol inflamatorio que es producida como un precursor inactivo denominado prointerleuquina $1 \beta$ (pro-IL-1 $\beta$ ). De forma similar, el complejo inflamasoma también está implicado en la escisión y activación de la interleucina 18 (IL-18), citoquina con actividad pro-aterogénica.

\section{Estructura del inflamasoma}

Varios miembros de la familia NLR, como NLRP1, NLRC4, NLRP6 y NLRP12, están de alguna manera implicados en la respuesta inmune al formar complejos multiproteicos de tipo inflamasoma, pero es el NLRP3 el más estudiado y en el que centraremos nuestro estudio [14]. Este inflamasoma está estructurado de la siguiente manera: un componente NLR, una proteína ASC (Apoptosis-associated speck-like protein containing a CARD) y la pro-caspasa-1. El componente NLR contiene en su extremo C-terminal un dominio rico en repeticiones de leucina (LRR), el cual está implicado en el reconocimiento del ligando y en mantener al NLR en estado inactivo; un dominio central de unión a nucleótidos NACHT (NOD) altamente conservado y un dominio N-terminal de tipo pirina (PYD). A través de este dominio piridina, el receptor NLR activo es capaz de unirse a la proteína ASC, la cual contiene un dominio homólogo PYD y uno de reclutamiento de caspasa (CARD) para la asociación con la pro-caspasa-1 [10].

\section{Activación de los inflamasomas}

Los componentes de los distintos inflamasomas se pueden activar mediante diferentes señales de peligro. El inflamasoma NLRP3, como se ha mencionado anteriormente, es activado por DAMP y PAMP y está por lo tanto implicado en la defensa del huésped [10]. Sus mecanismos de activación, sin embargo, son diversos. En primer lugar, es necesaria la señal desencadenada por los lipopolisacáridos (LPS), que

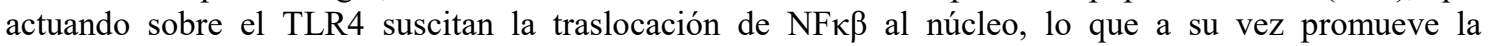
transcripción de la pro-IL-1 $\beta$ y del propio NLRP3. Posteriormente, son necesarias señales que permitan el ensamblaje del inflamasoma para su activación propiamente. Entre estas señales destaca el ATP, que al unirse a un receptor de membrana P2X7 permite el eflujo de potasio desde la célula, lo que activa el ensamblaje del inflamasoma [15]. Por otro lado, la generación de ROS (especies reactivas de oxígeno) mitocondrial también dispararía el ensamblaje de este inflamasoma. Finalmente, una tercera señal sería la desestabilización lisosomal con posterior ruptura del lisosoma y liberación de catepsina $\mathrm{B}$, siendo esta última la que activaría la formación del complejo [10]. En este último caso centraremos nuestra atención, puesto que es el mecanismo implicado en la activación del inflamasoma en la aterosclerosis: los macrófagos reclutados en la placa de ateroma fagocitan a los cristales de colesterol, pero un exceso de 
ello puede desestabilizar a los lisosomas causando su ruptura y liberación de catepsina $\mathrm{B}$, desencadenando la activación del inflamasoma NLRP3 [12].

\section{NLRP3 como diana terapéutica}

El inflamasoma NLRP3 es el encargado de la activación y posterior liberación de dos citoquinas de carácter pro-aterogénico: IL-1 $\beta$ e IL-18. Teniendo en cuenta el rol principal de este complejo NLRP3 en la respuesta inflamatoria en aterosclerosis, el objetivo de este trabajo se centró en la búsqueda de una molécula que antagonizase el efecto del receptor NLRP3 en la activación del inflamasoma y la liberación de IL-1 $\beta$ e IL-18. Con este fin, se diseñó un estudio de base meramente teórica que incluyó: un análisis mediante HTS, un cribado mediante un ensayo in vitro y una evaluación de los compuestos seleccionados mediante un ensayo in vivo.

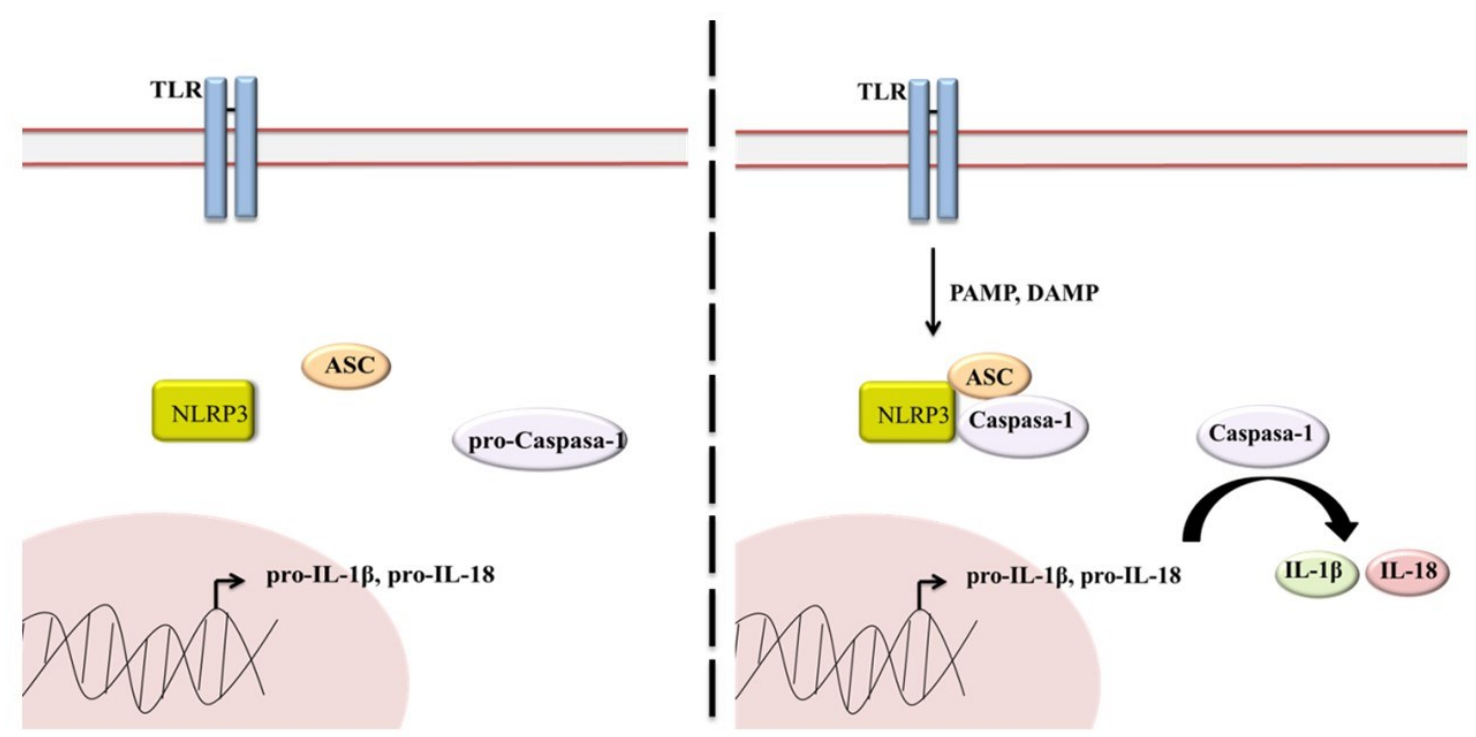

Figura 1 - Modelo de activación y funcionamiento del inflamasoma NLRP3. Las señales de peligro celular, entre ellas los DAMP o PAMP, inducen la asociación de los componentes del inflamasoma NLRP3: el receptor NLRP3, la proteína ASC y la pro-Caspasa-1. La formación del complejo inflamasoma conduce a la activación de la pro-Caspasa-1, la cual se escinde y transforma en Caspasa1. La Caspasa-1, a su vez, es capaz de inducir la escisión y activación de las interleucinas proinflamatorias y pro-aterogénicas IL-1 $\beta$ e IL-18.

\section{Materiales y Métodos}

HTS

Para testar grandes colecciones de compuestos químicos se emplea el cribado farmacológico denominado HTS. Esta técnica consiste en diseñar un ensayo que permita analizar los compuestos aplicando un determinado criterio de selección, con el fin de identificar moléculas biológicamente activas. Los compuestos seleccionados, que cumplan con los requisitos establecidos, reciben el nombre de hit. En el presente estudio, se diseñó un ensayo HTS en base a la inhibición farmacológica de nuestra diana, el inflamasoma NLRP3, con el fin de seleccionar los hits que puedan actuar en una posible estrategia terapéutica frente a la aterosclerosis. Para ello, se utilizó la técnica de Transferencia de Energía por Resonancia de Fluorescencia, también conocida como FRET, empleando las proteínas fluorescentes mCherry2 y EGFP [16]. Así, se procedió a elaborar un doble ensayo FRET en células HEK293T: en una primera fase, se buscaron compuestos que inhiban la unión del receptor NLRP3 con la proteína ASC. En una segunda fase, se seleccionaron aquellos compuestos que se unan específicamente al NLRP3 sin unirse a ASC. Con este fin, se propuso un segundo FRET a partir de los compuestos que superaron el primer ensayo para detectar una interacción positiva entre las proteínas ASC y AIM2.

Para los ensayos FRET, se elaboró un clonaje con la secuencia de la proteína ASC en el vector pcDNA3EGFP (Addgene), y las secuencias correspondientes a las proteínas NLRP3 y AIM2 en el vector mCherry2-N1 (Addgene). El producto de estos clonajes se corresponde con las siguientes proteínas de fusión: ASC-EGFP, NLRP3-mCherry2 y AIM2-mCherry2 (Figura 2). De esta forma, se co-transfectaron las células HEK293T empleando el reactivo lipofectamina con los plásmidos que codifican las proteínas de fusión ASC-EGFP y NLRP3-mCherry2. En paralelo, se diseñó una segunda co-transfección de células HEK293T con los plásmidos que codifican las proteínas de fusión ASC-EGFP y AIM2-mCherry2. Posteriormente, ambas variantes de células co-transfectadas se cultivaron en placas de 1536 pocillos, añadiendo en cada pocillo un compuesto de la quimioteca. A continuación, se procedió a la activación de 
los correspondientes inflamasomas: para el inflamosoma NLRP3 se empleó un tratamiento con nigericina, mientras que para el inflamosoma AIM2 se infectaron las células con virus Vaccina.

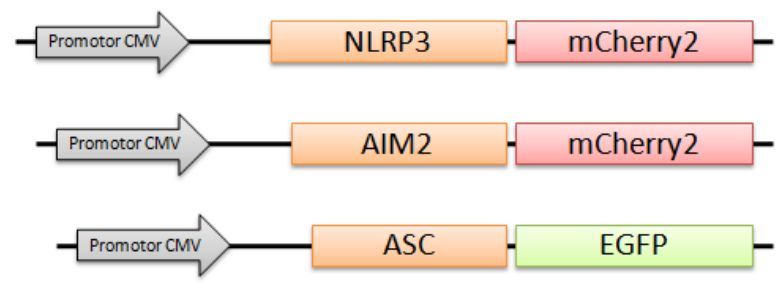

Figura 2 - Secuencia de las proteínas de fusión generadas tras el clonaje de las proteínas NLRP3, ASC y AIM2 en los vectores pcDNA3-EGFP y mCherry2-N1, que contienen las proteínas fluorescentes EGFP y mCherry2.

Por último, el análisis de las placas se diseñó empleando el lector multi-modo de microplacas para HTS PHERAstar ${ }^{\circledR}$ FSX (BMGa Labtech). En concreto, se utilizó el ensayo TR-FRET, que consiste en un FRET en el cual se miden las longitudes de onda emitidas a lo largo del tiempo. Así, las células cotransfectadas y tratadas se excitaron a una longitud de onda correspondiente a la excitación de la proteína $\operatorname{EGFP}(\lambda: 485 \mathrm{~nm})$, recogiéndose dos emisiones de luz: la emisión de la proteína fluorescente EGFP $(\lambda$ : $500-520 \mathrm{~nm})$ y la emisión de la proteína fluorescente mCherry2 $(\lambda: 600-620 \mathrm{~nm})$ (Figura 3).

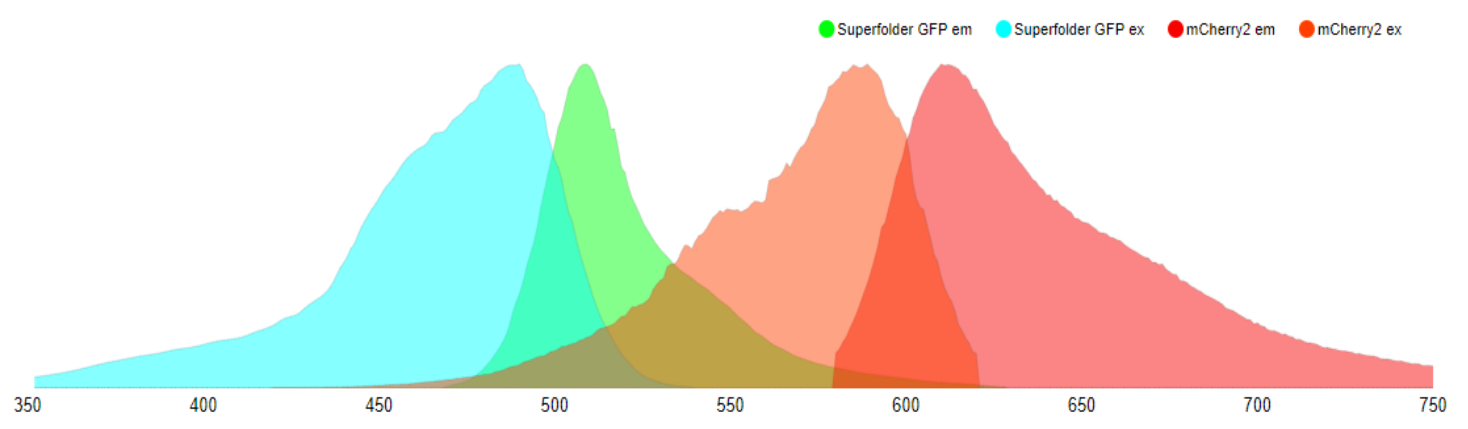

Figura 3 - Espectros de excitación y emisión de las proteínas fluorescentes empleadas en el ensayo FRET: EGFP y mCherry2. Para que este ensayo FRET sea efectivo, el espectro de emisión de la proteína EGFP debe solaparse con el espectro de excitación de la segunda proteína fluorescente, la proteína mCherry2.

\section{Estudio in vitro}

Con el fin de descartar los hits que no son útiles en la inhibición del inflamasoma NLRP3 y obtener leads o cabezas de serie, se diseñó un estudio in vitro que contemplaba los siguientes cribados: determinación de la liberación de IL-1 $\beta$ e IL-18, ensayo de viabilidad celular y ensayo de efectividad y potencia. Para el diseño de estos ensayos, fueron necesarias dos variantes de la línea celular de monocitos humanos THP1: THP1-defNLRP3 y THP1-nullNLRP3 (InvivoGen). La variante THP1-defNLRP3 presenta una baja actividad del NLRP3 y actividad normal del ASC y la pro-caspasa 1, mientras que la variante THP1nullNLRP3 presenta sobreexpresión de NLRP3, ASC y pro-caspasa 1, por lo que ambas líneas celulares resultaron óptimas para este estudio.

Para determinar la liberación de IL-1 $\beta$ e IL-18 en células THP1-defNLRP3 y THP1-nullNLRP3, se aplicó un tratamiento en dos fases con inductores de estos marcadores inflamatorios [17]. En primer lugar, con el objetivo de inducir la expresión de la pro IL-1 $\beta$ y la pro IL-18 mediante la vía NF-KB, se adicionaron moléculas de lipopolisacárido (LPS) al medio de cultivo y se tomó cierta cantidad de dicho medio para su análisis. Posteriormente, se procedió a la adición de cristales de colesterol para inducir la expresión de la IL-1 $\beta$ y la IL-18 a través del inflamasoma NLRP3, tomándose del mismo modo parte del medio de cultivo para su posterior análisis. En paralelo, se aplicó un segundo tratamiento a las células THP1nullNLRP3, que consistió en la adición de los compuestos hits al medio, previamente al uso de los cristales de colesterol, manteniendo todos los pasos anteriormente descritos. Este segundo tratamiento permitió analizar el efecto de los hits en la síntesis de ambas interleucinas (IL-1 $\beta$ y la IL-18). Las muestras, recogidas durante ambos tratamientos, se emplearon en la determinación de los niveles de IL$1 \beta$ e IL-18 humanas liberadas al medio empleando kits comerciales de ELISA (Thermo Fisher). Los compuestos hits que superasen dicho cribado se sometieron a un ensayo MTT (Innoprot) en células THP1-nullNLRP3 para verificar que los valores de las concentraciones de las interleucinas (IL-1 $\beta$ e IL18) en el medio de cultivo se deben al efecto del compuesto y no a la muerte celular. Finalmente, se propuso un ensayo de efectividad y potencia en células THP1-nullNLRP3. Para ello, se determinó la concentración de IL-1 $\beta$ e IL-18 en el medio de cultivo por técnica ELISA siguiendo el protocolo previamente descrito. En este caso, se emplean los compuestos que superen los cribados anteriores a 
diferentes concentraciones, y se construye con ello una curva dosis-respuesta. Esta curva dosis-respuesta representa el efecto en la liberación de las interleucinas respecto a la dosis de cada uno de nuestros hits, teniendo en cuenta que la potencia es la dosis a la que el compuesto alcanza la eficacia máxima, y que la eficacia es la respuesta terapéutica máxima.

\section{Estudio in vivo}

Para la valoración de aquellos compuestos seleccionados como lead, se diseñó un estudio in vivo empleando como modelo de aterosclerosis ratones macho C57BL/6 con una deleción génica global de la apoproteína E, de 6 semanas de edad, alimentados con dieta estándar. La deficiencia de la proteína ApoE impide la captación de lipoproteínas a nivel hepático, principalmente quilomicrones (QM) y lipoproteínas de muy baja densidad (VLDL), lo que genera un cuadro de hiperlipidemia, desarrollo de placas ateroscleróticas y lesiones de carácter inflamatorio [18]. Los ratones se dividieron en un grupo control no tratado y diversos grupos de tratamiento con administración de los compuestos establecidos como leads. Además, se incorporó un grupo adicional al que se le administró el fármaco Canakinumab, con la doble finalidad de desarrollar un estudio de superioridad de fármacos. Los compuestos se administraron por vía intraperitoneal, dos veces por semana, durante 10 semanas, tras lo cual se procedió al sacrificio del animal.

Se obtuvo mediante técnica de disección la arteria aorta desde su nacimiento, inmediatamente superior al anillo valvular aórtico, y por extracción, sangre periférica mediante punción cardiaca. La sangre periférica se empleó en la determinación de niveles de IL-1 $\beta$ e IL-18 mediante técnica de ELISA, así como en la determinación de un análisis general (perfil lipídico, función hepática, función renal). Las lesiones ateroscleróticas se analizaron en tejido aórtico mediante tinción Sudán IV, colorante que permite determinar el área y extensión de las placas ateroscleróticas. Adicionalmente, la luz del vaso y el CORE lipídico (parámetro que indica el rango que representa la proporción de lípidos respecto a la extensión de la placa) se determinaron mediante un estudio histológico del tejido aórtico con tinción tricrómica de Masson. Los resultados se analizaron digitalmente empleando el software Image J. Por último, el perfil inflamatorio se determinó por técnicas inmunohistoquímicas en cortes transversales del tejido aórtico, analizando los niveles de los siguientes marcadores pro-inflamatorios: CD68, VCAM-1, P-selectina y Eselectina [19].

\section{Resultados Esperados}

\section{HTS}

Del ensayo HTS propuesto en este estudio se esperaría obtener un listado de compuestos que inhiban específicamente la asociación entre el receptor NLRP3 y la proteína ASC, componentes principales del inflamasoma NLRP3 cuya interacción implica su activación. Este ensayo HTS se diseñó empleando la técnica FRET, que consiste en una interacción que ocurre solo a muy corta distancia entre dos estados de excitación electrónica de dos moléculas fluorescentes en el que la longitud de onda de emisión de una de ellas coincide con la excitación de la otra. En el presente estudio se emplearon proteínas de fusión compuestas por sustratos fluorescentes (EGFP y mCherry2) y las moléculas diana (NLRP3, ASC, AIM2). Con estas proteínas de fusión se diseñó un doble ensayo FRET que permite analizar el efecto de los compuestos de nuestra quimioteca en la interacción de estas proteínas.

Para esta selección HTS, se recogerían las emisiones de luz de los diferentes pocillos de las placas en las que se cultivaron las células HEK293T co-transfectadas con los correspondientes plásmidos de fusión y tratadas con los compuestos de la quimioteca. Si la asociación entre las proteínas de fusión tiene lugar, la excitación de la proteína EGFP sería transmitida a la proteína mCherry2, y esta emitiría a una longitud de onda concreta. Por el contrario, si la asociación entre las proteínas se pierde, se esperaría detectar solo la emisión correspondiente a la proteína EGFP. De esta forma, en una primera fase se analizaría la interacción entre las proteínas de fusión ASC-EGFP y NLRP3-mCherry2, y se seleccionarían los compuestos que aportasen una mayor señal en el canal de emisión de EGFP ( $\lambda$ : 500-520nm), puesto que estarían impidiendo esta unión y por tanto inhibiendo la activación del inflamasoma NLRP3. Utilizando estos compuestos seleccionados, en la segunda fase de este doble FRET se analizaría la asociación entre las proteínas de fusión ASC-EGFP y AIM2-mCherry2. En este caso, se elegirían los compuestos que producen una mayor señal en el canal de mCherry2 ( $\lambda$ : 600-620nm), ya que permiten la unión de ASC y AIM2. Con ello se comprobaría que los compuestos no bloquean a la proteína ASC. De este modo, los compuestos finales o hits serían moléculas que se unen específicamente al receptor NLRP3 e interfieren en su interacción con la proteína ASC. Al unirse específicamente al receptor NLRP3, se evitarían posibles efectos secundarios que surgirían si se inhibiesen otros inflamasomas en los que interviene la proteína ASC. Dado que ambos ensayos se diseñaron en células HEK293T, los compuestos elegidos además deberían ser capaces de atravesar la membrana plasmática. 


\section{Estudio in vitro}

El análisis de la liberación de marcadores inflamatorios (IL-1 $\beta$ e IL-18), generados como productos de la activación del inflamasoma NLRP3, se propuso mediante un ensayo in vitro en células THP1-defNLRP3 y THP1-nullNLRP3 empleando inductores como el LPS y los cristales de colesterol. Así, la concentración de interleucinas IL-1 $\beta$ e IL-18 detectada tras la adición del LPS se correspondería con los niveles basales, mientras que, tras la adición de los cristales de colesterol estos niveles se asumirían directamente como respuesta a la activación del inflamasoma NLRP3. Dada la baja actividad del NLRP3 en células THP1-defNLRP3, tras el tratamiento con cristales de colesterol no se esperaría encontrar diferencias en la concentración de interleucinas con respecto a la concentración basal. Por el contrario, sí se esperaría obtener un aumento de la concentración de IL-1 $\beta$ e IL-18 respecto a la concentración basal en células THP1-nullNLRP3. Evaluando el efecto de los hits en la liberación de IL-1 $\beta$ e IL-18, se seleccionarían aquellos compuestos que al añadir cristales de colesterol no hayan permitido el incremento de los niveles de estas interleucinas con respecto a la concentración basal. De este modo, se elegirían los hits que en células THP1-nullNLRP3 conduzcan a valores mínimos de IL-1 $\beta$ e IL-18 y comparables a los obtenidos en células THP1-defNLRP3. De acuerdo al criterio de selección empleado, se obtendría como resultado solo aquellos hits que bloqueasen la actividad del inflamasoma NLRP3 de manera específica.

Adicionalmente, los resultados obtenidos del ensayo de viabilidad en células THP1-nullNLRP3 proporcionarían una selección de los hits efectivos que a su vez no provocasen toxicidad celular, y así se verificaría que la falta de incremento en las concentraciones de interleucinas se debe al efecto del compuesto y no a la muerte celular. Por último, a través del ensayo de potencia y efectividad, se seleccionarían los compuestos (leads) más potentes y con la mayor respuesta terapéutica. En este caso, la máxima respuesta terapéutica se correspondería con la mínima concentración de interleucinas observada tras la adición de los cristales de colesterol. Gracias a la curva dosis-respuesta, se determinaría la dosis de compuesto a la que alcanza la respuesta máxima, y así se seleccionarían los leads de mayor eficacia, denominados aleatoriamente P3RM-1, P3RM-2 y P3RM-3.

\section{Estudio in vivo}

En el presente estudio propuesto a nivel in vivo, se emplearon ratones ApoE (-/) como modelo aterosclerótico para evaluar el efecto de nuestros leads o cabezas de serie. El papel de la proteína ApoE es el de interaccionar con el receptor LDL, localizado en la membrana hepática, e inducir la captación de lipoproteínas, principalmente QM y VLDL provenientes del intestino. Estas lipoproteínas son las que portan la mayor cantidad de colesterol, de forma que una deficiencia global de ApoE conduce a la imposibilidad de captación de estas partículas, y con ello a situaciones de hiperlipidemia. Además, la ApoE es una proteína implicada en inflamación, de forma que con este modelo se generan placas ateroescleróticas más avanzadas y lesiones con un mayor componente inflamatorio [18].

En los resultados que se esperarían obtener de este estudio in vivo, aquellos compuestos leads (P3RM-1, P3RM-2, P3RM-3) que inhiban específicamente el inflamasoma NLRP3, bloquearían la vía de síntesis de IL-1 $\beta$ e IL-18 a través de Caspasa 1, de forma que debería observarse una disminución de los niveles de estas interleucinas en sangre periférica en aquellos ratones tratados respecto al control. Esta disminución, además, debería ser superior respecto a la inducida por el fármaco Canakinumab. Al descender los niveles de IL-1 $\beta$ e IL-18, se observaría una menor gravedad en las lesiones o placas ateroscleróticas en aquellos ratones tratados con nuestros compuestos leads. Los resultados esperados incluirían un descenso de la extensión y el área de las placas, determinados en aorta. De igual modo, debería ser observable un aumento de la luz del vaso y una diminución del CORE lipídico en animales tratados. Un menor CORE lipídico (proporción de lípidos respecto a la extensión de la placa) reflejaría una menor inestabilidad de las placas ateroscleróticas, lo que implicaría probabilidades más bajas de desprendimiento de ésta y, por ende, de evento cardíaco o cerebrovascular. Por tanto, la disminución del CORE lipídico indicaría indirectamente un mejor pronóstico.

Respecto a las moléculas inflamatorias analizadas por inmunohistoquímica, debería detectarse una disminución en los niveles de CD68, indicador de la infiltración de macrófagos, en animales tratados respecto a sus controles. Igualmente, se observaría un descenso en los niveles de VCAM-1, E-selectina y P-selectina. Estos marcadores son las principales moléculas de activación del endotelio que intervienen en el reclutamiento y la extravasación de linfocitos y macrófagos, cuya expresión está mediada por citoquinas del tipo IL-1 $\beta$. Por tanto, estos resultados indicarían una menor respuesta inflamatoria debido a la interferencia en la liberación de citoquinas pro-inflamatorias IL-1 $\beta$ e IL-18, consiguiente al antagonismo del inflamasoma NLRP3. Así, la disminución en la respuesta inflamatoria podría mejorar el estado aterosclerótico y el pronóstico del paciente, dada la creciente relevancia que está tomando el componente inflamatorio en esta enfermedad. 


\section{Conclusiones}

Los datos aportados por el estudio Cantos en relación a la inhibición de la IL-1 $\beta$ mediante el tratamiento con Canakinumab han sido revolucionarios tras mostrar el papel protagónico de la inflamación en la fisiopatología de la enfermedad aterosclerótica, a la vez que han abierto nuevos campos de investigación en el área cardiovascular. El presente estudio plantea una nueva estrategia que, de llegar a superar el plano teórico, podría mejorar las alternativas terapéuticas existentes hasta el momento. Además, conociendo los mecanismos fisiopatológicos de muchas enfermedades cardiovasculares y no cardiovasculares, en donde la inflamación, y en particular las citoquinas dependientes de la vía del inflamasoma NLRP3 juegan un rol fundamental, se estaría en condiciones de pensar que de llegar a una posible molécula que inhiba específicamente al receptor NLRP3, su aplicación cubriría un amplio espectro clínico aún mayor que el tratado en este artículo.

\section{Conflicto de intereses}

Los autores no declaran conflicto de intereses.

\section{Referencias}

1. Ibanez, B., James, S., Agewall, S., Antunes, M.J., Bucciarelli-Ducci, C., Bueno, H., Caforio, A.L.P., Crea, F., Goudevenos, J.A., Halvorsen, S., Hindricks, G., Kastrati, A., Lenzen, M.J., Prescott, E., Roffi, M., Valgimigli, M., Varenhorst, C., Vranckx, P., Widimský, P. and ESC Scientific Document Group. 2018. 2017 ESC Guidelines for the management of acute myocardial infarction in patients presenting with ST-segment elevation: The Task Force for the management of acute myocardial infarction in patients presenting with ST-segment elevation of the European Society of Cardiology (ESC). Eur Heart J. 39(2):119-77.

2. Sacks, F.M., Pfeffer, M.A., Moye, L.A., Rouleau, J.L., Rutherford, J.D., Cole, T.G., Brown, L., Warnica, J.W., Arnold, J.M., Wun, C.C., Davis, B.R. and Braunwald, E. 1996. The effect of pravastatin on coronary events after myocardial infarction in patients with average cholesterol levels. Cholesterol and Recurrent Events Trial investigators. N Engl J Med. 335(14):1001-9

3. Downs, J.R., Beere, P.A., Whitney, E., Clearfield, M., Weis, S., Rochen, J., Stein, E.A., Shapiro, D.R., Langendorfer, A. and Gotto, A.M.Jr. 1997. Design \& Rationale of the Air Force/Texas Coronary Atherosclerosis Prevention Study (AFCAPS/TexCAPS). Am J Cardiol. 80(3):287-93

4. Albert, M.A., Glynn, R.J., Fonseca, F.A., Lorenzatti, A.J., Ferdinand, K.C., MacFadyen, J.G. and Ridker, P.M. 2011. Race, ethnicity, and the efficacy of rosuvastatin in primary prevention: The Justification for the Use of Statins in Prevention: an Intervention Trial Evaluating Rosuvastatin (JUPITER) trial. Am Heart J. 162(1):106-14. e2

5. Nicholls, S.J., Borgman, M., Nissen. S.E., Raichlen, J.S., Ballantyne, C., Barter, P., Chapman, M.J., Erbel, R. and Libby, P. 2011. Impact of statins on progression of atherosclerosis: rationale and design of SATURN (Study of Coronary Atheroma by InTravascular Ultrasound: effect of Rosuvastatin versus AtorvastatiN). Curr Med Res Opin. 27(6):1119-29

6. Cannon CP1, Braunwald E, McCabe CH, Rader DJ, Rouleau JL, Belder R, Joyal SV, Hill KA, Pfeffer MA, Skene AM; Pravastatin or Atorvastatin Evaluation and Infection TherapyThrombolysis in Myocardial Infarction 22 Investigators. 2004. Intensive versus moderate lipid lowering with statins after acute coronary syndromes. N Engl J Med. 2004 Apr 8;350(15):1495-504

7. de Lemos, J.A., Blazing, M.A., Wiviott, S.D., Lewis, E.F., Fox, K.A., White, H.D., Rouleau, J.L., Pedersen, T.R., Gardner, L.H., Mukherjee, R., Ramsey, K.E., Palmisano, J., Bilheimer, D.W., Pfeffer, M.A., Califf, R.M. and Braunwald, E., Investigators. Early intensive vs a delayed conservative simvastatin strategy in patients with acute coronary syndromes: phase $Z$ of the A to $\mathrm{Z}$ trial. JAMA. 292(11):1307-16

8. Nissen, S.E., Tuzcu, E.M., Schoenhagen, P., Brown, B.G., Ganz, P., Vogel, R.A., Crowe, T., Howard, G., Cooper, C.J., Brodie, B., Grines, C.L. and De Maria, A.N. REVERSAL Investigators. 2004. Effect of intensive compared with moderate lipid-lowering therapy on progression of coronary atherosclerosis: a randomized controlled trial. JAMA. 291(9):1071-80

9. Ridker, P.M., Libby, P., MacFadyen, J.G., Thuren, T., Ballantyne, C., Fonseca, F., Koenig, W., Shimokawa, H., Everett, B.M. and Glynn, R.J. 2018. Modulation of the interleukin-6 signalling pathway and incidence rates of atherosclerotic events and all-cause mortality: analyses from the Canakinumab Anti-Inflammatory Thrombosis Outcomes Study (CANTOS). Eur Heart J. 39(38):3499-3507

10. Karasawa, T. and Takahashi, M. 2017. The crystal-induced activation of NLRP3 inflammasomes in atherosclerosis. Inflamm Regen. 37: 18 
11. Duewell, P., Kono, H., Rayner, K.J., Sirois, C.M., Vladimer, G., Bauernfeind, F.G., Abela, G.S., Franchi, L., Nuñez, G., Schnurr, M., Espevik, T., Lien, E., Fitzgerald, K.A., Rock, K.L., Moore, K.J., Wright, S.D., Hornung, V. and Latz, E. 2010. NLRP3 inflammasomes are required for atherogenesis and activated by cholesterol crystals. Nature. 464(7293):1357-61

12. Karasawa, T. and Takahashi, M. 2017. Role of NLRP3 Inflammasomes in Atherosclerosis. J Atheroscler Thromb. 24(5): 443-51

13. Martinon, F., Burns, K. and Tschopp. J. 2002. The Inflammasome: A Molecular Platform Triggering Activation of Inflammatory Caspases and Processing of proIL- $\beta$. Mol Cell. 10(2):417-26

14. Abderrazak, A, Couchie, D., Mahmood, D.F., Elhage, R., Vindis, C., Laffargue, M., Matéo, V., Büchele, B., Ayala, M.R., El Gaafary, M., Syrovets, T., Slimane, M.N., Friguet, B., Fulop, T., Simmet, T., El Hadri, K. and Rouis, M.. 2015. Anti-inflammatory and antiatherogenic effects of the NLRP3 inflammasome inhibitor arglabin in ApoE2.Ki mice fed a high-fat diet. Circulation. 131(12):1061-70

15. Redondo-Castro, E., Faust, D., Fox, S., Baldwin, A.G., Osborne, S., Haley, M.J., Karran, E., Nuthall, H., Atkinson, P.J., Dawson, L.A., Routledge, C., Allan, S.M., Freeman, S., Brownlees, J. and Brough, D. 2018. Development of a characterised tool kit for the interrogation of NLRP3 inflammasome-dependent responses. Sci Rep. 8(1):5667

16. Day, R. N. and Davidson, M. W. 2012. Fluorescent proteins for FRET microscopy: monitoring protein interactions in living cells. Bioessays. 34(5):341-350

17. Abderrazak, A., Couchie, D., Mahmood, D. F., Elhage, R., Vindis, C., Laffargue, M., Matéo, V., Büchele, B., Ayala, M.R., El Gaafary, M., Syrovets, T., Slimane, M.N., Friguet, B., Fulop, T., Simmet, T., El Hadri, K. and Rouis, M. 2015. Anti-inflammatory and antiatherogenic effects of the NLRP3 inflammasome inhibitor Arglabin in ApoE2.Ki mice fed a high-fat diet. Circulation. 131(12): 1061-70

18. Emini Veseli, B., Perrotta, P., De Meyer, G.R.A., Roth, L., Van der Donckt, C., Martinet, W. and De Meyer, G.R.Y. 2017. Animal models of atherosclerosis. Eur J Pharmacol. 816(1):3-13

19. Bhaskar, V., Yin, J., Mirza, A.M., Phan, D., Vanegas, S., Issafras, H., Michelson, K., Hunter, J.J. and Kantak, S.S. 2011. Monoclonal antibodies targeting IL-1 beta reduce biomarkers of atherosclerosis in vitro and inhibit atherosclerotic plaque formation in Apolipoprotein E-deficient mice. Atherosclerosis. 216(1):313-20 